\title{
Quantum properties of the parametric amplifier with and without pumping field fluctuations
}

\author{
Faisal A. A. El-Orany, ${ }^{1}$ J.Peřina, ${ }^{1}$ and M. Sebawe Abdalla ${ }^{2}$ \\ 1 Department of Optics, Palacký University, \\ 17. listopadu 50, 772 07 Olomouc, Czech Republic \\ ${ }^{2}$ Mathematics Department, College of Science, \\ King Saud University, P.O. Box 2455, Riyadh 11451, Saudi Arabia
}

(Dated: October 10, 2018)

\begin{abstract}
The parametric amplifier with and without the pumping fluctuations of coupling function is considered when the fields are initially prepared in coherent light. The pumping fluctuations are assumed to be normally distributed with time-dependent variance. The effects of antibunching and anticorrelation of photons on the photon distribution, correlation between modes and factorial moments are demonstrated. A possible enhancement of photon antibunching for certain values of initial mean photon numbers is shown and discussed. We have shown also that new states (called modified squeezed vacuum states or even thermal states) can be generated from such an interaction. Further, we have demonstrated that the sum photon-number distribution can exhibit collapses and revivals in the photon-number domain somewhat similar to those known in the Jaynes-Cummings model.

PACS numbers: 42.50Dv,42.60.Gd
\end{abstract}

\section{INTRODUCTION}

Quadratic parametric processes have been considered since 1961, when a quantum-mechanical model of the parametric amplification and frequency conversion, treating the pump field classically and neglecting the losses, had been proposed in [1]. Among these processes parametric amplifier takes a considerable interest since degenerate and nondegenerate parametric amplifiers can perfectly provide single-mode and two-mode squeezing (squeezed light has less noise than a coherent light in one of the field quadratures and can exhibit a number of features having no classical analogue). Further, parametric amplifier has been employed in experiments. For example, the fourth-order interference effects arise when pairs of photons produced in parametric amplifier are injected into Michelson interferometers [2]. The second-order interference is observed in the superposition of signal photons from two coherently pumped parametric amplifiers when the paths of the idler photons are aligned [3]. 
It is well-known that the interaction of light with matter changes the statistical properties of light. The nature of the change depends on the kind of interaction process and on the statistical properties of the incident electromagnetic field. An investigation of the statistical properties of the nondegenerate parametric amplifier (without losses) has been performed using different techniques, such as Heisenberg technique [4] and Schrödinger technique [5]. The main attention in these articles has been paid to the single mode case, i.e. only one mode (signal or idler) is detected. The anticorrelation in this model is an interesting effect [6], where the variance of the photon number is less than the average photon number and the photocounting distribution becomes narrower than the corresponding Poisson distribution for a coherent state with the same mean photon number. These nonclassical phenomena are established for certain values of the phase of incident fields. On the other hand, the quantum statistical properties of the parametric amplification and generation including losses in the medium have been studied by means of the generalized Fokker-Planck equation for the distribution related to the antinormal ordering [7] showing that the normal counting generating function decomposes into the product of two functions which are the generating functions for the superposition of coherent and chaotic fields and that the outgoing field exhibits the anticorrelation effect. Further, it is convenient mentioning that the photon counting distribution together with the waiting-time distribution techniques have been used to analyze the nonclassical effects for light beams generated by the lossy degenerate optical parametric amplifier [8].

Finally, we refer to parametric processes with stochastic pumping [9, 10, 11, 12, 13, 14] modelled by means of a stochastic differential equations. One can find the total photon number, as a function of time, of frequency up-conversion process has a turning point and the antibunching effect is predicted regardless of the mean photon numbers available in the process. Also the effect of pumping fluctuations are considered in [12, 13, 14], where the observed sub-Poissonian behaviour is progressively degraded as the level of fluctuations in the pumping radiation increases. This means that the pump fluctuations play a similar role as reservoir, where the interaction with the environment leads to the destruction of the nonclassical effect, i.e. to the coherent energy extraction from the quantum mechanical system.

In the present paper we study the quantum properties of the parametric amplifier with the classical pumping by considering the pump fluctuations in the system. More illustratively, the lossless effective Hamiltonian [12, 13, 14] controlling the system is 


$$
\hat{H}=\hbar \omega_{1} \hat{a}_{1}^{\dagger} \hat{a}_{1}+\hbar \omega_{2} \hat{a}_{2}^{\dagger} \hat{a}_{2}-\hbar g\left[\hat{a}_{1} \hat{a}_{2} \exp (i \omega t-i \phi)+\text { h.c }\right]
$$

where $\hat{a}_{j}, j=1,2$, are the annihilation operators designated to the signal and idler mode, respectively; $\omega_{j}$ are the natural frequencies of oscillations of the uncoupled modes with $\omega=\omega_{1}+\omega_{2}$ (assuming the resonance frequency condition holds); $\phi$ is the initial phase of the pump and h.c. is the Hermitian conjugate; $g$ is the real coupling constant (phase matching is assumed) and we assume $g=g_{0}+\varepsilon g_{1}$, that is the coupling constant consists of two parts: $g_{0}$ is the deterministic part and $g_{1}$ is the correction part (i.e. $\left|g_{0}\right|>>\left|\varepsilon g_{1}\right|$ ), and $\varepsilon$ is a real fluctuating quantity obeying time-dependent Gaussian distribution described by

$$
P(\varepsilon, t)=\frac{1}{\sqrt{2 \pi \sigma(t)}} \exp \left[-\frac{\varepsilon^{2}}{2 \sigma(t)}\right],
$$

where the standard deviation is $\sigma(t)=\sigma_{0}[1-\exp (-\mu t)], \sigma_{0}$ is the maximum standard deviation and $\mu$ is a finite real parameter. This is the difference from what has been done earlier [12, 13, 14], where the standard deviation is time independent. In this way pump fluctuations are taken into account. Indeed, time-dependent standard deviation can lead to the enhancement of nonclassical effects compared to the time-independent case, as we shall see later. For any observable $\hat{F}(\varepsilon, t)$, the average processs will be performed as follows:

$$
\langle\langle\hat{F}(\varepsilon, t)\rangle\rangle=\int_{-\infty}^{\infty}\langle\hat{F}(\varepsilon, t)\rangle_{\mathrm{f}} P(\varepsilon, t) d \varepsilon
$$

where the two angle brackets means that there are two average operations, one is the average (expectation value) over the initial states of the field $\langle. .\rangle_{\mathrm{f}}$ and the other is the average over the stochastic parameter $\varepsilon$ using the distribution (2) in the sense described on the right-hand side of (3). Such an averaging process will be frequently used in this article.

We should stress here that the model will be treated generally for two cases with and without the pump fluctuations where the comparison is instructive. Also we give new insight into the possibility to generate nonclassical light based on the change of intensity of initial incident fields which has not been considered earlier [7, 12, 13, 14].

For completeness, the solution of the Heisenberg's equations for the Hamiltonian (1) is well known 


$$
\begin{aligned}
& \hat{a}_{1}(t)=\exp \left(-i \omega_{1} t\right)\left[\hat{a}_{1}(0) \cosh (g t)+i \hat{a}_{2}^{\dagger}(0) \sinh (g t) \exp (i \phi)\right], \\
& \hat{a}_{2}(t)=\exp \left(-i \omega_{2} t\right)\left[\hat{a}_{2}(0) \cosh (g t)+i \hat{a}_{1}^{\dagger}(0) \sinh (g t) \exp (i \phi)\right] .
\end{aligned}
$$

The small term $|\varepsilon| g_{1}<<\left|g_{0}\right|$ is considered as a small perturbation and the standard deviation $\sigma(t)$ is assumed as a slowly varying quantity compared to the variations in (4).

This article is organized as follows: In section 2 the basic equations and relations such as anticorrelation functions, reduced factorial moments and sum photon-number distribution are given when the modes are initially prepared in coherent light. In section 3 discussion of the results is performed and the conclusions are summed in section 4 .

\section{BASIC RELATIONS AND EQUATIONS}

As it is well known light fields for which the $P$ representation is a well-behaved distribution cannot exhibit nonclassical features [15, 16]. P-function for the single mode as output from parametric amplifier (1) is well defined, because in the parametric systems the evolution broadens the single mode $P$ distribution due to the spontaneous pump photon decay [17]. This also increases the radius of the Wigner contour compared to the initial one [18] and reflects that there is no (single mode) nonclassical behaviour. For this reason we focus our attention on the behaviour of the compound modes when the modes are initially prepared in coherent states.

Now, cross-correlation may be used to describe anticorrelation between modes, which may cause that the variance of the photon number is less than the average of the photon number giving then antibunching; it can be measured by detecting single modes separately by two photodetectors and correlating their outputs. Cross-correlation between mode 1 and mode 2 is controlled by

$$
\left\langle: \triangle W_{1} \triangle W_{2}:\right\rangle=\left\langle\left\langle: \hat{a}_{1}^{\dagger}(t) \hat{a}_{1}(t) \hat{a}_{2}^{\dagger}(t) \hat{a}_{2}(t):\right\rangle\right\rangle-\left\langle\left\langle\hat{a}_{1}^{\dagger}(t) \hat{a}_{1}(t)\right\rangle\left\langle\hat{a}_{2}^{\dagger}(t) \hat{a}_{2}(t)\right\rangle\right\rangle,
$$

where $W_{j}=\hat{a}_{j}^{\dagger}(t) \hat{a}_{j}(t), j=1,2$, and : : denotes the normally ordered operator, i.e. creation operators $\hat{a}_{j}^{\dagger}$ are to the left of annihilation operators $\hat{a}_{j}$; the double angle brackets in the righthand side has the same meaning as before. 
For input coherent light $\left(\left|\alpha_{1}, \alpha_{2}\right\rangle\right)$ equation (5) together with (4) reduce to [7, 12, 13, 14 ]

$$
\begin{array}{r}
\left\langle: \triangle W_{1} \triangle W_{2}:\right\rangle=\left\langle\frac{1}{4}\left(2\left|\alpha_{1}\right|^{2}+2\left|\alpha_{2}\right|^{2}+1\right) \sinh ^{2}(2 g t)\right. \\
\left.-\frac{1}{2}\left|\alpha_{1}\right|\left|\alpha_{2}\right| \sinh (4 g t) \sin \psi\right\rangle, \\
=\frac{1}{8}\left(2\left|\alpha_{1}\right|^{2}+2\left|\alpha_{2}\right|^{2}+1\right)\left\{\cosh \left(4 g_{0} t\right) \exp \left[8 \sigma(t) g_{1}^{2} t^{2}\right]-1\right\} \\
-\frac{1}{2}\left|\alpha_{1}\right|\left|\alpha_{2}\right| \sinh \left(4 g_{0} t\right) \exp \left[8 \sigma(t) g_{1}^{2} t^{2}\right] \sin \psi,
\end{array}
$$

where $\alpha_{j}=\left|\alpha_{j}\right| \exp \left(i \phi_{j}\right), j=1,2\left(\left|\alpha_{j}\right|\right.$ and $\phi_{j}$ are the amplitude and the phase of the initial coherent $j$ th mode (signal or idler)), and $\psi=\phi_{1}+\phi_{2}-\phi$ represents the difference between the phase-sum of the initial signal and idler modes and the initial phase of the pump, which is the quantity determining the phase mismatch. In (6) and in the following we have performed the average with respect to the initial coherent states and the average over stochastic pumping field, which is indicated by the angle brackets (in (7) the integration over $\varepsilon$ has been carried out using the distribution (21) after prescription (3) ).

We proceed by writing down the formulae of the reduced factorial moments and sum photonnumber distribution for compound modes; the details of the derivation for these relations can be found in [12, 13], respectively as

$$
\begin{aligned}
& \left\langle W^{k}\right\rangle=\left\langle\sum_{l=0}^{k}\left(\begin{array}{l}
k \\
l
\end{array}\right) \lambda_{1}^{k-l} \lambda_{2}^{l} \mathrm{~L}_{k-l}\left(-\frac{A_{1}}{\lambda_{1}}\right) \mathrm{L}_{l}\left(-\frac{A_{2}}{\lambda_{2}}\right)\right\rangle, \\
& =\sum_{l=0}^{k} \sum_{l_{1}=0}^{k-l} \sum_{l_{2}=0}^{l} \sum_{l_{3}=0}^{k-l_{1}-l_{2}}\left(\begin{array}{l}
k \\
l
\end{array}\right)\left(\begin{array}{c}
k-l \\
l_{1}
\end{array}\right)\left(\begin{array}{l}
l \\
l_{2}
\end{array}\right) \\
& \left(\begin{array}{c}
k-l_{1}-l_{2} \\
l_{3}
\end{array}\right) \frac{(-1)^{k+l_{3}-l-l_{1}}(k-l) ! l ! h_{1}^{l_{1}} h_{2}^{l_{2}}}{l_{1} ! l_{2} !} \\
& \times\left(\frac{1}{2}\right)^{k-l_{1}-l_{2}} \exp _{A_{1}}\left[2 g_{0} t\left(l-l_{1}-l_{3}\right)+2 \sigma(t) g_{1}^{2} t^{2}\left(l-l_{1}-l_{3}\right)^{2}\right], \\
& P(n, t)=\left\langle\frac{\exp \left[-\frac{A_{1}}{\left(1+\lambda_{1}\right)}-\frac{A_{2}}{\left(1+\lambda_{2}\right)}\right]}{\left(1+\lambda_{1}\right)\left(1+\lambda_{2}\right)} \sum_{l=0}^{n} \frac{\lambda_{1}^{n-l} \lambda_{2}^{l}}{(n-l) ! l !}\left(1+\lambda_{1}\right)^{l-n}\left(1+\lambda_{2}\right)^{-l}\right. \\
& \left.\times \mathrm{L}_{n-l}\left[-\frac{A_{1}}{\lambda_{1}\left(1+\lambda_{1}\right)}\right] \mathrm{L}_{l}\left[-\frac{A_{2}}{\lambda_{2}\left(1+\lambda_{2}\right)}\right]\right\rangle, \\
& =\sum_{l=0}^{n} \sum_{l_{1}=0}^{n-l} \sum_{l_{2}=0}^{l} \sum_{l_{3}=0}^{n-l_{1}-l_{2}} \sum_{l_{4}=0}^{\infty}\left(\begin{array}{c}
n-l \\
l_{1}
\end{array}\right)\left(\begin{array}{l}
l \\
l_{2}
\end{array}\right)\left(\begin{array}{c}
n-l_{1}-l_{2} \\
l_{3}
\end{array}\right) 4^{l_{1}+l_{2}+1} \\
& \times \frac{(-1)^{n+l_{3}+l_{4}-l-l_{1}}\left(n+l_{1}+l_{2}+l_{4}+1\right) ! h_{1}^{l_{1}} h_{2}^{l_{2}}}{l_{1} ! l_{2} ! l_{4} !\left(n+l_{1}+l_{2}+1\right) !} \exp \left[-\Gamma_{0}\left(t, l_{j}\right)+\frac{1}{2} \Gamma^{2}\left(t, l_{j}\right) \sigma(t)\right],
\end{aligned}
$$


where

$$
\begin{aligned}
& \lambda_{1}=\frac{1}{2}[\exp (-2 g t)-1], \quad \lambda_{2}=\frac{1}{2}[\exp (2 g t)-1], \quad A_{1}=h_{1} \exp (-2 g t), \\
& A_{2}=h_{2} \exp (2 g t), \quad h_{1}=\frac{1}{2}\left(\left|\alpha_{1}\right|^{2}+\left|\alpha_{2}\right|^{2}+2\left|\alpha_{1}\right|\left|\alpha_{2}\right| \sin \psi\right), \\
& h_{2}=\frac{1}{2}\left(\left|\alpha_{1}\right|^{2}+\left|\alpha_{2}\right|^{2}-2\left|\alpha_{1}\right|\left|\alpha_{2}\right| \sin \psi\right), \\
& \Gamma_{1}(t)=-\frac{4\left(h_{1}-h_{2}\right) t g_{1} \exp \left(-2 g_{0} t\right)}{\left[1+\exp \left(-2 g_{0} t\right)\right]^{2}}, \quad \Gamma_{2}(t)=2 h_{1}-\frac{2\left(h_{1}-h_{2}\right)}{1+\exp \left(-2 g_{0} t\right)}, \\
& \Gamma\left(t, l_{j}\right)=\Gamma_{1}(t)+2 g_{1} t\left(l_{1}+l_{2}+l_{3}+l_{4}+1\right), \\
& \Gamma_{0}\left(t, l_{j}\right)=\Gamma_{2}(t)+2 g_{0} t\left(l_{1}+l_{2}+l_{3}+l_{4}+1\right)
\end{aligned}
$$

and $\mathrm{L}_{n}($.$) is the Laguerre polynomial of order n$; as before the angle brackets in (8) and (10) represent the average over the stochastic pumping variable $\varepsilon$. For simplicity we have dropped the time $t$ from the coefficients $A_{j}$ and $\lambda_{j}$. The quantities $A_{j}$ and $\lambda_{j}$ play the role of the mean numbers of coherent photons and mean numbers of chaotic photons, respectively. The transition from (8) to (9) as well as from (10) to (11) has been done by carrying out the integration over the stochastic variable. It is reasonable mentioning that the expressions (91) and (11) are different from those in [12, 13], where the average over the initial complex field amplitudes is taken there and consequently additional summations have appeared. When performing this integration the following expressions have been used in the treatment of the exponential factor for the sum photon-number distribution:

$$
\begin{aligned}
& \frac{A_{1}}{1+\lambda_{1}}+\frac{A_{2}}{1+\lambda_{2}}=2 h_{1}-2\left(h_{1}-h_{2}\right)[1+\exp (-2 g t)]^{-1} \\
& =2 h_{1}-2\left(h_{1}-h_{2}\right) \sum_{l=0}^{\infty}(-1)^{l} \exp \left(-2 l g_{0} t\right)\left[1-2 g_{1} t l \varepsilon+O\left(\varepsilon^{2}\right)\right] \\
& =2 h_{1}-2\left(h_{1}-h_{2}\right)\left[\frac{1}{1+\exp \left(-2 g_{0} t\right)}+\frac{2 g_{1} t l \varepsilon \exp \left(-2 g_{0} t\right)}{\left[1+\exp \left(-2 g_{0} t\right)\right]^{2}}\right]
\end{aligned}
$$

where in these relations $g$ is replaced by its value, and $O\left(\varepsilon^{2}\right)$ includes terms of the power equal or higher than $\varepsilon^{2}$, which have been neglected in the last step since $\varepsilon$ is small. In spite of this the neglection is physically sensible and necessary for performing the average over the parameter $\varepsilon$, it leads to a problem that the distribution (11) does not exist as an ordinary well behaved function. Consequently additional treatments may be used to avoid this difficulty. Therefore, if 
the propagation time through the crystal, which is also the interaction time, is sufficiently short together with the fact that the contribution of the correction part (the term involving $g_{1}$ ) must be small compared with the main contribution, some modification of the correction term can be performed. For example we can approximate $g_{1}^{2} t^{2} l_{4}^{2}$ by $g_{1}^{2} t^{2}\left(l_{1}+l_{2}+l_{3}+1\right) l_{4}$ in the exponential term. The choice of the factor $\left(l_{1}+l_{2}+l_{3}+1\right)$ is to compensate the large values of $l_{4}$ as much as possible since $l_{j}, j=1,2,3$ are the indices of the summations (cf. (11)). It is important to point out for a short time interaction (as well as finite values of $g_{1}$ and $\sigma_{0}$ ) and finite values of $l_{4}$ that the contribution of $\Gamma_{2}^{2}(t)$ is considerably small compared with that of $\Gamma_{0}(t)$. The validity of this approximation can be checked numerically. Now the sum photon-number distribution (11) becomes well behaved function, as it should be, and having the form

$$
\begin{aligned}
& P(n, t)=\sum_{l=0}^{n} \sum_{l_{1}=0}^{n-l} \sum_{l_{2}=0}^{l} \sum_{l_{3}=0}^{n-l_{1}-l_{2}}\left(\begin{array}{c}
n-l \\
l_{1}
\end{array}\right)\left(\begin{array}{c}
l \\
l_{2}
\end{array}\right)\left(\begin{array}{c}
n-l_{1}-l_{2} \\
l_{3}
\end{array}\right) \frac{(-1)^{n+l_{3}-l-l_{1}} h_{1}^{l_{1}} h_{2}^{l_{2}} 4_{1}^{l_{1}+l_{2}+1}}{l_{1} l_{2} !} \\
& \times\left\{1+\exp \left[2 \sigma(t) g_{1}^{2} t^{2}\left(1+\frac{\Gamma_{1}(t)}{g_{1} t}+3\left(l_{1}+l_{2}+l_{3}+1\right)\right)-2 t g_{0}\right]\right\}^{-\left(n+l_{1}+l_{2}+2\right)} \\
& \times \exp \left\{-2 t g_{0}\left(l_{1}+l_{2}+l_{3}+1\right)-\Gamma_{2}(t)+\frac{1}{2} \sigma(t)\left[\Gamma_{1}(t)+2 t g_{1}\left(l_{1}+l_{2}+l_{3}+1\right)\right]^{2}\right\} .
\end{aligned}
$$

One can easily check that when $g_{1} \rightarrow 0$, all equations for fluctuating pumping reduce to the nonfluctuating ones.

In the following we investigate the behaviour of the system on the basis of the results of the present section.

\section{DISCUSSION OF THE RESULTS}

The discussion of the results of the model under consideration will be performed through two parts. In the first part we give an investigation for both the anticorrelation between modes and the reduced factorial moments. However, the second part is devoted to the analysis of the sum photon-number distribution. Also in this part we develop new states and discuss some of its properties.

\section{A. Anticorrelations and reduced factorial moments}

We start our discussion in this part by analyzing the anticorrelations between modes in the system, which can show some interesting features. For usual parametric amplifier (i.e. $g=g_{0}$ ), one can easily check that the extreme values of (6) are established at $\psi= \pm \pi / 2$, i.e. the maximum 


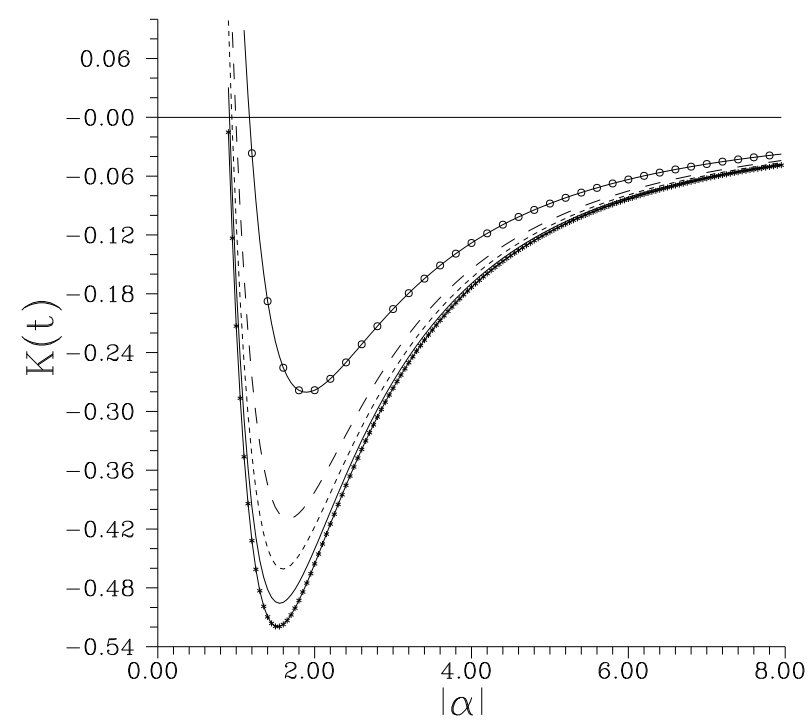

FIG. 1: The normalized correlation of fluctuations between modes $\left[K(t)=\left\langle: \triangle W_{1} \triangle W_{2}:\right\rangle /\left\langle W_{1}\right\rangle\left\langle W_{2}\right\rangle-1\right]$, against $|\alpha|$ for $\psi=\pi / 2, t=0.5, g_{0}=1, \mu=1, g_{1}=0.6$, for the time-dependent fluctuating pumping with $\sigma_{0}=0.1$ (solid curve), 0.5 (long-dashed curve) and for the time-independent fluctuating pumping with $\sigma_{0}=0.1$ (short-dashed curve), 0.5 (circle-centered curve). The star-centered curve is corresponding to that of the usual parametric amplifier and the straight line is the bound of anticorrelation.

value is at $\psi=-\pi / 2$ and minimum value at $\psi=\pi / 2$. Careful examination shows that it is always positive when $\psi=-\pi / 2$, i.e. the behaviour is always classical. The maximal nonclassical effect can appear when $\psi=\pi / 2$. In other words, the anticorrelation is observable as a result of coupling of modes. Further, considering that $\alpha_{1}=\alpha_{1}=\alpha, \quad t>0$, and $\psi=\pi / 2$, one can easily obtain that anticorrelation occurs if the relation (6) is negative and this provides

$$
|\alpha|>\frac{1}{2} \exp \left(g_{0} t\right) \sqrt{\sinh \left(2 g_{0} t\right)}
$$

if the equality holds this expression means that coherent light can be recovered. This relation gives the range of $|\alpha|$ over which the anticorrelation can be obtained. The right hand side of (15) is monotonically increasing function in the course of time and then the nonclassical effect can be realized for a certain time interval after switching on the interaction. It can be shown that the maximum value of anticorrelation between modes is $-\frac{1}{4} \exp \left(4 g_{0} t\right) \sinh ^{2}\left(2 g_{0} t\right)$, which occurs at $|\alpha|=\frac{1}{2} \exp \left(2 g_{0} t\right) \sinh \left(4 g_{0} t\right)$. Also the maximum value of correlation establishes at $|\alpha|=0$. Indeed, all these informations are clear in Figs. 1,2, where the normalized forms of both correlation between modes and reduced factorial moments have been plotted against $|\alpha|$, respectively. From these figures one can see that the anticorrelation reflects itself in the moments of reduced factorial 


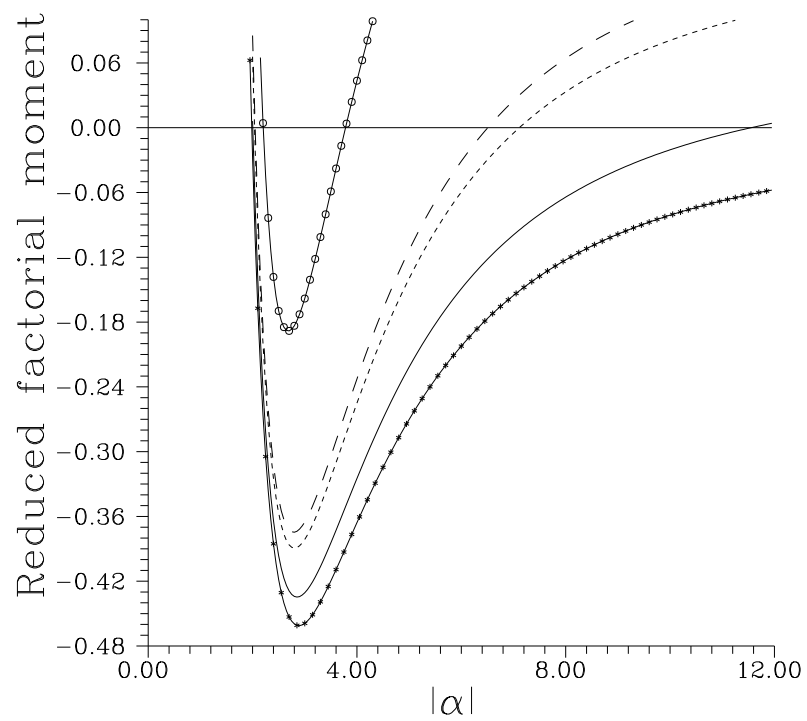

FIG. 2: The reduced factorial moments $\left(\left\langle W^{k}\right\rangle /\langle W\rangle^{k}-1\right)$ against $|\alpha|$ when $k=5$ for $\psi=\pi / 2, t=0.5, g_{0}=$ $1, \mu=1, g_{1}=0.4$, for the time-dependent fluctuating pumping with $\sigma_{0}=0.1$ (solid curve), 0.3 (long-dashed curve) and for the time-independent fluctuating pumping with $\sigma_{0}=0.1$ (short-dashed curve), 0.3 (circlecentered curve). The star-centered curve is corresponding to that of the usual parametric amplifier and the straight line is the bound of antibunching.

moment. In general, the behaviour of these quantities is smooth where, e.g. for reduced factorial moment, all curves start from the bunching values going to the antibunching ones as the initial mean photon number increases until they reach a maximum, then they start again to decrease the antibunching values and eventually become stable for a large domain of $|\alpha|$. The most effective antibunching can arise for the usual parametric amplifier as expected (see star-centered curves). When the pumping field fluctuations are considered, the degradation of the nonclassical effect occurs (see Figs. 1 and 2). From these figures, the comparison between the solid and short-dashed curves as well as between the long-dashed and circle-centered curves shows that the nonclassical effects for the standard deviation in time-dependent case are more pronounced than those for the time-independent case. Further, one can see also that as the value of $\sigma_{0}$ increases, the amount of antibunching decreases.

Now we would like to conclude this part by giving an example when the interaction time is short which is relevant for practical situation of propagating waves in nonlinear media. We discuss this point only for the normalized correlation between modes where similar arguments can be given for the reduced factorial moments. This has been displayed in Fig. 3 for the shown values of the parameters. We note when the standard deviation is time-dependent, the behaviour of the normalized correlation of fluctuations between modes is the same for all chosen values and 


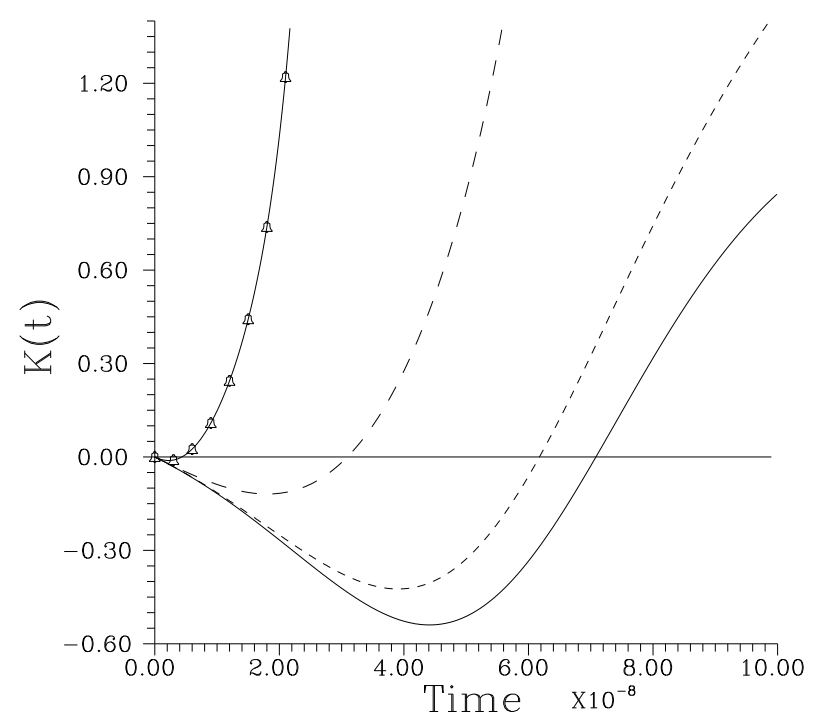

FIG. 3: The normalized correlation of fluctuations between modes for $\psi=\pi / 2,\left|\alpha_{j}\right|^{2}=2, \mu=1, g_{0}=10^{7} s^{-1}$, for the time-dependent fluctuating pumping (solid curve) and for the time-independent fluctuating pumping. Short-dashed curve is for $g_{1}=10^{6} s^{-1}, \sigma_{0}=10$, long-dashed curve for $g_{1}=10^{7} s^{-1}, \sigma_{0}=1$, bell-centered curve for $g_{1}=10^{7} s^{-1}, \sigma_{0}=10$. The solid curve is for the usual parametric amplifier as well as for the time-dependent fluctuating pumping.

coincides with the behaviour of the usual parametric amplifier, which gives the most effective anticorrelation values (see solid curve in Fig. 3). This seems to be related to the short interaction time. Also we noted that the anticorrelation nature becomes less pronounced as $g_{0}$ (or interaction time $t$ ) increases, where the amplification of radiation can occur and this is connected with an increase in chaotic noise [20]. Indeed, the regime of anticorrelation or antibunching is related to the damping of the field [7, 20]. In conclusion one can control the intensity of initial fields, phases, and interaction time in such a way that we can get maximum antibunching effect. This phenomenon can be observed by detecting both the modes 1 and 2 simultaneously beyond the nonlinear medium. It is reasonable to mention that the sum of mode noises of $W=W_{1}+W_{2}$ can be less than modulus of negative values of $2\left\langle: \triangle W_{1} \triangle W_{2}:\right\rangle$, i.e. antibunching can occur if $\left\langle:(\triangle W)^{2}:\right\rangle<0\left(\left\langle: \triangle W_{1} \triangle W_{2}:\right\rangle<0\right)$, which we have also verified. Such situation cannot occur in classical physics and this leads to the nonexistence of the Glauber-Sudarshan quasidistribution. Such quiet light may be applied in optical signal processing and optical communications. 


\section{B. Sum photon-number distribution}

Firstly at $\alpha_{j}=0, \quad j=1,2$, for usual parametric amplifier, equation (10) for the sum photonnumber distribution reduces to

$$
P(n, t)=\frac{[-\tanh (g t)]^{n}}{\cosh ^{2}(g t)} \sum_{l=0}^{n}(-1)^{l} .
$$

This expression shows that only even terms survive and this is nonclassical effect. Moreover, such photon-number distribution indicates that the system collapses to produce the state

$$
\begin{aligned}
& |\Psi(t)\rangle=\frac{1}{\cosh (g t)} \sum_{l=0}^{\infty}[\tanh (g t)]^{l} \exp (i l \chi)|2 l\rangle, \\
& =\frac{1}{2 \cosh (g t)} \sum_{l=0}^{\infty}[\tanh (g t) \exp (i \chi)]^{\frac{l}{2}}\left[1+(-1)^{l}\right]|l\rangle,
\end{aligned}
$$

where $\chi$ is a relevant phase. In fact, state (17) is quite different from both squeezed vacuum states 21] and single-mode of two-mode squeezed vacuum states [19]. These two states are determined respectively by

$$
\begin{gathered}
|\Psi(t)\rangle_{1}=\frac{1}{\sqrt{\cosh (g t)}} \sum_{l=0}^{\infty}\left[\frac{\tanh (g t)}{2}\right]^{l} \frac{\sqrt{(2 l) !}}{l !}|2 l\rangle, \\
\rho_{\mathrm{I}}(t)=\frac{1}{\cosh ^{2}(g t)} \sum_{l=0}^{\infty}[\tanh (g t)]^{2 l}|l\rangle\langle l|,
\end{gathered}
$$

where $\rho_{\mathrm{I}}(t)$ is the reduced density operator representing the single-mode case of two-mode squeezed vacuum states.

The comparison of the three states (17)-(19) is instructive. One can find that states (17) are completely different from states (19), where the latter is the state usually associated with chaotic Bose-Einstein statistics [19], i.e. it cannot exhibit nonclassical effects. However, the former can exhibit nonclassical effects due to the superposition principle. Actually, states (17) can be written in the form of even thermal states by tracing over the phase through the relation

$$
\begin{aligned}
& \hat{\rho}_{\text {eth }}=\frac{1}{2 \pi} \int_{0}^{2 \pi}|\Psi(t)\rangle\langle\Psi(t)| d \chi \\
& =\frac{1}{\bar{n}+1} \sum_{l=0}^{\infty}\left(\frac{\bar{n}}{\bar{n}+1}\right)^{2 l}|2 l\rangle\langle 2 l|,
\end{aligned}
$$

where $\bar{n}=\sinh ^{2}(g t)$; in this case $\bar{n}$ represents the mean number of thermal photons and $\hat{\rho}_{\text {eth }}$ means the density matrix of the even thermal states. On the other hand, states (17) and (18) are different in their structures but they can have similar nonclassical effects. For instance, both states can have 


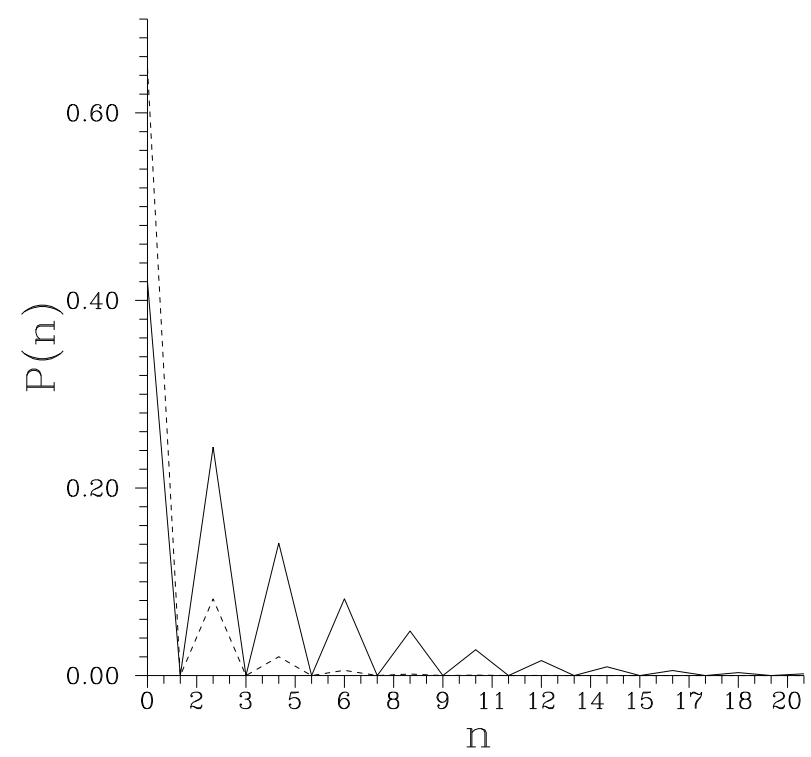

FIG. 4: The photon-number distribution of squeezed vacuum states (dashed-curve) and of modified squeezed vacuum states (solid curve) against the number of photons $n$ for $t=0.5, g_{0}=2, g_{1}=0$.

pairwise oscillation in the photon-number distribution but with different amplitudes, also they can display quadratures squeezing rather than sub-Poissonian statistics (which can be easily checked). So that we shall call state (17) as a modified squeezed vacuum state (or even thermal state). In Fig. 4 we have plotted the photon-number distribution for squeezed vacuum and modified squeezed vacuum states for comparison. From this figure it is clear that the oscillations in the photon-number distribution of state (17) are more pronounced than those of (18). These oscillations are purely quantum effect without classical analogue. The origin of these pairwise oscillations in (18) is two-photon nature of the squeeze operator [21] and they have been interpreted by means of the interference in phase space [22] or in the framework of the generalized superposition of coherent fields and negative quantum noise [23]. Nevertheless, for the state (17) the origin of the oscillatory behaviour in the photon-number distribution is the quantum interference between the components of the state (see second line of (17)) in phase space. In fact, such a type of interference is quite different from the interference of the Schrödinger cat states [24]. To make this point clear we may analyze the behaviour of Wigner $(W)$ function for modified squeezed vacuum states since this function is informative and sensitive to the interference in phase space. Applying standard technique, the $W$-function of (17) is

$$
W(z)=\frac{1}{\pi \cosh (g t)}\left\{\exp \left[-g t-2|z|^{2} e^{-2 g t}\right]+\exp \left[g t-2|z|^{2} e^{2 g t}\right]\right\}
$$

where $z=x+i y$. 


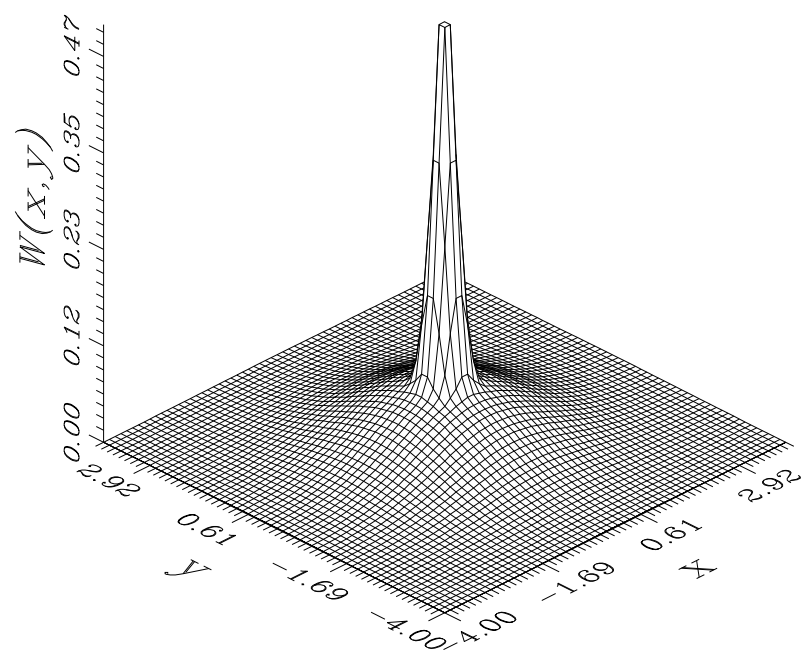

FIG. 5: The $W$-function of modified squeezed vacuum against $\operatorname{Re} z=x, \operatorname{Im} z=y$ for the same situation as Fig. 4.

This relation shows that the $W$-function cannot exhibit negative values and its contour in phase space is symmetric in contrast with that of squeezed states showing noise ellipse. Further, it contains two terms, the first one is arising from the statistical mixture and the second represents the interference between the components of the state (17). Consequently the behaviour of $W$-function here depends on the competition between these two terms where both have a Gaussian shape with center at the origin, however, the first term is always broader than the second one. This is clear in Fig. 5 where $W$-function given by (21) is plotted. In fact, such interference is responsible for the nonclassical effects of the modified squeezed vacuum states. Further, similar behaviour has also been seen for even binomial states [25], which contains a finite number of Fock basis states. Finally, it should be stressed here that states (17) cannot be produced by a unitary operator as the squeezed vacuum state. This is so becuase there is no Hermitian Hamiltonian from which the state (17) can be generated via a canonical transformation. To be more specific, substituting $|2 l\rangle=\frac{\hat{a}^{\dagger 2}}{(2 l) !}|2 l\rangle$ into the right-hand side of (17), one can find that the summation cannot be performed to obtain the required unitary operator and this is in contrast with (19). Further, the second line of (17) shows that this state represents a superposition of two like-phase-coherent states [26] and consequently it cannot be obtained from any unitary operation [27].

Now let us return to the sum photon-number distribution when $\alpha_{j} \neq 0, \quad j=1,2$. Actually we noted that it is in a rough agreement with the normalized correlation of fluctuations between modes. For instance, for the usual parametric amplifier and when $\psi=-\pi / 2, \quad t>0, P(n, t)$ almost exhibits single-peak structure broader than that of the corresponding coherent light with the same 

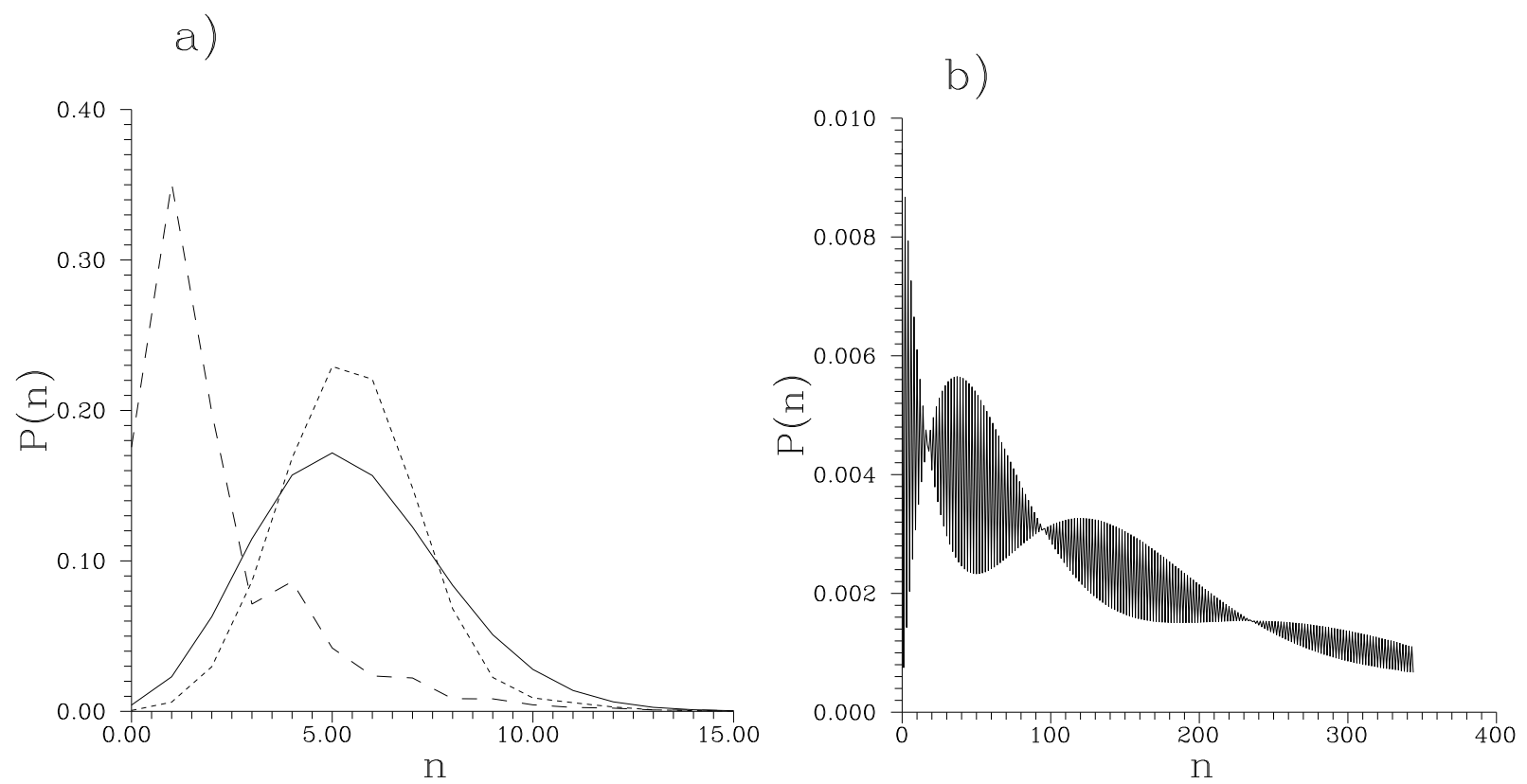

FIG. 6: The sum photon-number distribution against the number of photon $n$ for a) usual parametric amplifier when $\psi=\pi / 2, t=0.5, g_{1}=0, \mu=1,\left(g_{0},\left|\alpha_{j}\right|\right)=(1,2.5884)$ (short-dashed curve), $(1.5,0.87)$ (longdashed curve), the solid curve is for the corresponding Poissonian distribution with the same $\langle W(t)\rangle$; b) time-dependent fluctuating pumping when $\psi=\pi / 2, t=3 \times 10^{-4}, g_{1}=0.06, \sigma_{0}=0.02,\left(g_{0},\left|\alpha_{j}\right|\right)=\left(10^{4}, 2\right)$.

mean photon numbers. However, when $\psi=\pi / 2, P(n, t)$ can exhibit single-peak or oscillatory behaviour according to the relation between the incident mean photon numbers, coupling constant and the interaction time (see Figs. $6 \mathrm{a}$ and $\mathrm{b}$ for shown values of the parameters). In Fig. 6a $|\alpha|=$ $\frac{1}{2} \exp \left(2 g_{0} t\right) \sinh \left(4 g_{0} t\right)$, i.e. when the maximum antibunching effects occur in normalized correlation function between modes. One can observe that the distribution for this case (short-dashed curve) is narrower than that of the corresponding Poissonian distribution showing nonclassical effects. Similar behaviours have been seen for the values of the parameters satisfying inequality (15). On the other hand, when $|\alpha|=\frac{1}{2} \exp \left(g_{0} t\right) \sqrt{\sinh \left(2 g_{0} t\right)}-0.25$, i.e. the model exhibits always bunching as it is clear from (15). In this case the photon-number distribution can exhibit nonclassical oscillations (long-dashed curve), however, it is broader than that for Poissonian light (which is not included in the figure). The sum photon-number distributions of time-dependent and timeindependent fluctuating pump have a similar behaviour for short interaction time (see Fig. 6b). From this figure there are evident the large scale macroscopic oscillations in the behaviour of $P(n, t)$. To be more specific, $P(n, t)$ exhibits collapses and revivals similar to those familiar in the Jaynes-Cummings model (JCM) [28]; the former is in the photon number domain rather than the time domain. In fact, observations on the collapses and revivals of Rabi oscillations were 
reported [29]. Further, collapses and revivals in the JCM are showing the granular structure of the photon-number distribution, however, here they indicate the establishment of strong correlation between the signal and idler modes in the nonlinear crystal. Indeed, similar phenomenon has been seen for the photon-number distribution of single-mode [30] and two-mode [31] squeezed coherent states with complex squeeze and displacement parameters. For the two-mode case the phenomenon has been realized for the diagonal, and joint photon-number distribution having a fixed number of photons in one of the modes, however, the case displayed here (Fig. 6b) for the sum photon-number distribution is much richer than for the above distributions [31].

\section{CONCLUSIONS}

As was shown earlier [7, 12, 13] one can get antibunching from the system described by (1) at a short time and for specific choice of the parameters. Also such an effect can be obtained from parametric amplification process with quantum pumping [6] regardless of the initial phase condition. In this case the source of this effect is the quantization of the pumping. In this article we have analyzed the role of the initial mean photon numbers on generated nonclassical light (antibunched light) which has not been considered earlier [7, 12, 13, 14] so that the overall quantum efficiency for the effect can be enlarged. In other words, the degree of antibunching can be controlled depending on the initial mean photon numbers. Further, we have shown that considering time-dependent pump fluctuations, they decrease the rate of degradation of the nonclassical effects compared with the time-independent case. This is evident from the behaviour of the reduced factorial moments and the normalized correlation of fluctuations between modes. The behaviour of the sum photon-number distribution is in agreement with the behaviour of reduced factorial moments and normalized correlation of fluctuations between modes showing antibunching, however, in some situations they can display nonclassical oscillations including their collapses and revivals.

We have shown also that we can generate a modified squeezed vacuum states (or even thermal states) when the two-mode vacuum states evolve in the present interaction, thus detecting the sum photon-number distribution for outgoing light using photodetectors. These states have somewhat similar behaviours as squeezed vacuum states, however, the origin of the nonclassical effects for these states is in the quantum interference between components of the state. The photon-number distribution for such states exhibits more pronounced oscillations than those of squeezed vacuum states for the same chosen parameters. 


\section{Acknowledgement}

J. P. and F. A. A. E-O. aknowledge the partial support from the Projects VS96028, LN00A015 and Research Project CEZ: J 14/98 of Czech Ministry of Education and from the Project 202/00/0142 of Czech Grant Agency. One of us (M. S. A.) is grateful for the financial support from the Project Math 1418/19 of the Research Centre, College of Science, King Saud University.

\section{References}

[1] W. H. Louisell and A. Yariv, Phys. Rev. 124 (1961) 1646.

[2] P. G. Kwiat, W. A. Vareka, C. K. Hong, H. Nathel and R. Y. Chiao, Phys. Rev. A 41 (1990) 2910; Z. Y. Ou, X. Y. Zou, L. J. Wang and L. Mandel, Phys. Rev. Lett. 65 (1990) 321.

[3] X. Y. Zou, L. J. Wang and L. Mandel, Phys. Rev. Lett. 67 (1991) 318.

[4] B. R. Mollow and R. J. Glauber, Phys. Rev. 160 (1967) 1076; 1097.

[5] L. Mišta, Czech J. Phys. B 19 (1969) 443.

[6] L. Mišta and J. Peřina, Acta Phys. Pol. A 52(3) (1977) 425.

[7] L. Mišta and J. Peřina, Czech J. Phys. B 28 (1978) 392.

[8] R. Vyas and S. Singh, Phys. Rev. A 40 (1989) 5147; A. B. Dodson and R. Vyas, Phys. Rev. A 47 (1993) 3396.

[9] W. S. Kryszewski and J. Chrostowski, J. Phys. A 10 (1977) 261.

[10] S. K. Srinivasan and S. Udayabaskaran, Opt. Act. 26 (1979) 1535.

[11] W. J. Mielniczuk, Opt. Act. 26 (1979) 1115.

[12] V. Peřinová, Opt. Act. 28 (1981) 747.

[13] V. Peřinová and J. Peřina, Opt. Act. 28 (1981) 769.

[14] J. Peřina: Quantum Statistics of Linear and Nonlinear Optical Phenomena, 2nd edition (Kluwer, Dordrecht 1991).

[15] R. J. Glauber, Phys. Rev. 130 (1963) 2529.

[16] E. Schrödinger, Naturwiss. 14 (1926) 664.

[17] A. Bandilla and H.-H. Ritze, Opt. Commun. 34 (1980) 190.

[18] S. M. Barnett and P. L. Knight, J. Opt. Soc. Am. B 2 (1985) 467.

[19] S. M. Barnett and P. L. Knight, J. Mod. Opt. 34 (1987) 841.

[20] L. Mišta, V. Peřinová, J. Peřina and Z. Braunerová, Act. Phys. Pol. A 51 (1977) 739.

[21] H. P. Yuen, Phys. Rev. A 13 (1976) 2226. 
[22] W. Schleich and J. A. Wheeler, Nature 326 (1987) 574; W. Schleich and J. A. Wheeler, J. Opt. Soc. Am. B 4 (1987) 1715; W. Schleich, D. F. Walls and J. A. Wheeler, Phys. Rev. A 38 (1988) 1177.

[23] J. Peřina and J. Bajer, Phys. Rev. A 41 (1990) 516; J. Peřina, Z. Hradil and B. Jurčo, Quantum Optics and Fundamentals of Physics (Kluwer, Dordrecht 1994).

[24] E. Schrödinger, Nature 23 (1935) 44.

[25] M. S. Abdalla, M. H. Mahran and A.-S. F. Obada, J. Mod. Opt. 41 (1994) 1889; A.-S. F. Obada, M. H. Mahran, F. A. A. El-Orany and M. S. Abdalla, Int. J. Th. Phys. 35 (1996) 1393.

[26] J. H. Shapiro, S. R. Shepard and N. W. Wong, Phys. Rev. Lett. 62 (1991) 2377; J. H. Shapiro and S. R. Shepard, Phys. Rev. A 43 (1991) 3795.

[27] K. Wódkiewicz, P. L. Knight, S. J. Buckle and S. M. Barnett, Phys. Rev. A 35 (1987) 2567.

[28] E. T. Jaynes and F. Cummings, Proc. IEEE 51 (1963) 89; N. H. Eberly, N. B. Norozhny and J. J. Sanchez-Mondragon, Phys. Rev. Lett. 44 (1980) 1323.

[29] G. Rempe, H. Walther and N. Klein, Phys. Rev. Lett. 58 (1987) 353

[30] B. Dutta, N. Mukunda, R. Simon and A. Subramaniam, J. Opt. Soc. B 10 (1993) 253.

[31] M. Selvadoray, M. S. Kumar and R. Simon, Phys. Rev. A 49 (1994) 4957. 